\title{
Improving the Hip Fracture Surgical Consenting Process: A Single Centre Multi-Cycle Quality Improvement Process
}

Kirit Singh ( $\sim$ SinghK20@cardiff.ac.uk)

Cardiff University https://orcid.org/0000-0002-0419-5381

\section{Ali Assaf}

Withybush General Hospital

Morgan Bayley

Royal Gwent Hospital

Gordon Gillespie

Royal Gwent Hospital

\section{Research}

Keywords: Checklists, Control charts/Run charts, Reminders, Surgery

Posted Date: March 13th, 2020

DOI: https://doi.org/10.21203/rs.3.rs-17083/v1

License: (9) This work is licensed under a Creative Commons Attribution 4.0 International License. Read Full License

Version of Record: A version of this preprint was published at Patient Safety in Surgery on June 13th, 2020. See the published version at https://doi.org/10.1186/s13037-020-00252-8. 


\section{Abstract}

Background Hip fractures are a common acute trauma presentation that if requiring surgery, should be performed within 48 hours. Consenting the patient is an important element of the pathway from admission to operating theatre and errors can lead to delayed procedures, medico-legal exposure and may represent a loss of patient autonomy. The aim of this project was to evaluate areas for improvement in our consenting process for patients with capacity and trial new strategies to determine which achieved the greatest improvement.

Methods Following a baseline measurement $(n=24)$, deficiencies were noted in three key process measures of clarity of documentation, failure to record procedure-specific risks and not offering a copy of the consent form to the patient. Two quality improvement cycles then followed using targeted teaching for doctors performing the consenting process.

Results Cycle 1 ( $n=26)$ following targeted teaching demonstrated improved clarity, with a reduction in abbreviations from $38 \%$ to $20 \%$, while more than doubling the documentation of discussion of key procedure-specific risks from $31 \%$ to $72 \%$. More patients were offered a copy of their consent form, rising from $12 \%$ to $48 \%$. Cycle $2(n=24)$ saw the introduction of pre-printed "risk of procedure" stickers. Although clarity measures continued to improve, improvements in pre-procedure risk documentation remained broadly static while the number of forms being offered to patients starkly declined to $8 \%$.

Conclusions Our project would suggest that while pre-printed stickers can be useful memory aids, specific teaching on consenting produces the greatest benefit. The usage of such tools should therefore be limited, as adjuncts only to detailed training.

\section{Background}

Hip fractures are one of the most common presentations to emergency departments in the United Kingdom with 66,000 cases reported per year (1). This is a significant injury that carries a $6.9 \%$ mortality risk within one month and requires prompt management (1). Delay of surgical intervention (if indicated) beyond 48 hours is associated with increased death rates and complications such as pressure sores (2). Trauma \& Orthopaedics departments are therefore required to make the pathway to operation as efficient as possible to ensure that patients are operated on within this window.

A key element of this pathway is gaining the patient's consent for the procedure. Patients should have their treatment options explained to them, along with the benefits of any intervention, along with associated risks, burdens and side effects before then reaching a decision on whether to proceed (3). Commonly for acute trauma admissions, this consenting process is performed by the clerking doctor to prepare the patient for an imminent operation. However often the clerking doctor may not be the surgeon performing the procedure and can be a relatively junior grade who is working under significant time or work pressure (4). These pressures have been noted to result in poor documentation and missed 
elements of admission notes (5) which can render important documentation such as consent forms substandard or even invalid (4).

In order to reduce the variation in the admission and consenting process, several approaches have been evaluated, ranging from enhanced training to the use of procedure-specific proformas (6) and consent stickers with pre-printed risks (7). Although these have demonstrated benefit, their usage remains limited and best practice is still unknown. This quality improvement project aimed to assess our baseline consenting practice within a large 774-bed district general hospital while also reviewing the impact of ongoing improvement efforts such as teaching sessions and the use of proformas and consent stickers.

Reviews of consent documentation for common trauma procedures have found poor documentation of risks (8) often with a wide variety of complications listed for the same procedure in varying frequencies (9). These deficiencies in documentation have been attributed to not only the aforementioned work pressures but also poor overall awareness of local or national guidelines and standards (10), which is often exacerbated by a relatively rapid turnover of junior staff (rotating as frequently as every 4 months) (5). This is despite Department of Health and Royal College of Surgeons guidelines on consent that state the person obtaining consent should have clear knowledge of the procedure along with the potential risks and complications $(11,12)$.

Poor or incomplete documentation during the consent process denies the patient a full understanding of the procedure they are about to undergo, negatively impacting their decision making autonomy (13). This can also then subsequently expose hospitals and doctors to possible litigation (14), (15). Previous reviews of litigation relating to informed consent in the United States found errors in recording the risks of a procedure accounted for up to $70 \%$ of complaints alone (16). Furthermore, if consent documentation is of poor enough quality to be rendered entirely invalid, this can delay the operative pathway, potentially resulting in cancellation of procedures (4). In a time critical situation such as hip fractures, these unnecessary delays can therefore significantly negatively impact patient care and outcomes.

Set proformas/checklists for common presentations have been found to improve the documentation of important items which can commonly be missed (17). Checklists of essential pre-operative tasks have been used to improve the rate of completion of requested jobs (18) and their usage in surgical ward rounds has also been found to improve the quality of documentation (19). It has also been established that checklists can have a significant role in changing cultural practice and improving safety (20) with work by the Safe Surgery Saves Lives Study Group finding that a checklist was associated with concomitant reductions in both rates of death and complications in patients undergoing non-cardiac surgery (21)

Stickers have been used as part of a checklist, for documentation of ward rounds and demonstrated to be a simple and effective way of evidencing good practice against recommended standards (22). A quality improvement program in NHS Lanarkshire where the use of ward round stickers was audited found them to be a simple and effective safeguard to ensure basic aspects of care were not missed while maintaining standards (23). The benefits of this standardized approach subsequently led to the 
development of websites devoted to consent for specific procedures. The goal of these resources was to provide a uniform approach to documenting risks, and their use has been shown to produce an improvement in quality of consenting (24). Similar benefits were found when using pre-printed consent labels for consenting patients $(7,25)$.

However, these efforts are prone to abandonment when local champions of their use move on (26) and it is recognized that the usage of proformas often tails off over time for a variety of reasons (e.g. logistical issues with re-printing when initial supplies are depleted) (27). Use of pre-printed stickers for consent may also pose a medico-legal issue given the implications of recent Supreme Court judgements, who found in Montgomery vs Lanarkshire Health Board that pre-procedure risk documentation should not only be generic but also reviewed for significance to the individual patient (28). Other projects using stickers have attempted to address this by having suggestion boxes to advise the consenter of the legal obligation to pause and engage in discussion with the patient (7), but documenting this individualized analysis of risks remains a challenge.

\section{Method}

\section{Baseline Measurement}

Over a 4-week period in August 2017, consent forms for patients admitted as emergency trauma due to hip fracture were audited against Royal College of Surgeons Standards (add reference). Patients were included if deemed to have mental capacity to consent for a procedure and excluded if they did not. This was achieved by only selecting consent form documentation that was used for those people aged 16 and over and having capacity, or for those under 16 years who were determined to be Gillick competent (referred to locally as Consent Form 1 (29)). All other consent form types were not reviewed for the purposes of this project (such as those for paediatric cases or for those lacking capacity.)

All data on the forms were analysed, with special attention being given to three process measures of clarity of documentation, whether the listed risks were appropriate for the intervention and if a copy of the consent form had been given or offered to the patient. Evaluation of clarity focussed on if all the patient details had been filled in correctly, along with if the procedure details were correct including laterality and site of surgery and whether abbreviations had been used.

In this period, 24 consent forms for neck of femur fracture patients with capacity were identified. While $93 \%$ had accurate completion of patient details and documentation of the patient's agreement to consent, $38 \%$ of forms used abbreviations instead of fully writing out the procedure. Documentation of generic risks such as infection, bleeding and thromboembolic events was completed in $95 \%$ of reviewed forms. However, significant procedure-specific risks such as dislocation and leg length discrepancy were documented in just $31 \%$ of forms. Finally, only $12 \%$ of patients were offered or given a copy of their consent form. 


\section{Design}

Given the significant omissions in consent form documentation and the consenting process, it was clear that improvement was necessary. The majority of documentation had been completed by junior members of the team, over a period that represented their first four weeks within the speciality. It therefore was felt that to improve documentation of procedure-specific risks, further teaching would be necessary to improve their understanding of the operation and what significant complications could arise. Improved oversight was also to be implemented with ongoing senior clinician review of documentation to be performed on the morning ward round to identify any errors (such as abbreviations) or omissions. Recognizing also that junior staff were completing these documents often in time or work pressured environments, further memory aids were also reviewed and developed including pre-printed consent form stickers with all key procedure-specific risks included. These could then be directly applied to the generic consent forms that were currently in use.

\section{Strategy And Improvement Cycles}

Having determined that lack of experience in junior trainees was a key reason for poor documentation, interventions were planned to align with each rotation where new trainees would start working within the department. Practice would then be re-audited over another four-week period to determine the impact of any interventions made.

Improvement Cycle 1 (January 2018) - Teaching for junior colleagues was carried out, including a session at induction for newly rotated junior doctors on how to complete consent in accordance with Royal College of Surgeons guidelines (12). Other teaching sessions were also performed for the department as a whole, covering the most common risks of procedures performed for high frequency presentations. Alongside this, senior review of consent forms in the morning ward round to identify errors was implemented. However, errors still occurred with procedure-specific risks found to be absent in $28 \%$ of cases.

Improvement Cycle 2 (August 2018) - As part of an updated approach to managing hip fractures, a proforma was developed including pre-printed consent stickers with all risks (both generic and procedurespecific) included (example for intracapsular neck of femur fractures requiring arthroplasty shown in Fig. 1). These stickers were then to be used in conjunction with existing consent form documentation and act as a memory prompt for the doctor's discussions with the patient. A teaching session on the use of this form along with the key procedure-specific risks was given to junior staff at their induction as they began their rotation.

\section{Results}

Following the teaching interventions in cycle 1, re-audit of practice identified 26 consent forms suitable for analysis. Significant improvement was seen in reducing the usage of abbreviations, which fell from 
38-20\% and procedure detail documentation improved further from a baseline of $93-96 \%$. Improvement was also found in documentation of all risks improving from $66-81 \%$ and procedure-specific risks documented in $72 \%$ of analysed forms, more than doubling baseline measurements. More patients were also offered a copy of their consent forms, rising from $12-48 \%$.

With the introduction of the hip fracture proforma and consent stickers in cycle 2, a further 24 consent forms were evaluated (see Fig. 2 - summary runchart). Sustained improvements were seen in documentation of procedure details, rising to $100 \%$ completion on all forms sampled. Abbreviation usage continued to fall further, decreasing to $7 \%$. However, documentation of procedure-specific risks remained similar to the prior cycle at $67 \%$ while a significant deterioration was seen in patients being offered a copy of their consent form, falling to just $8 \%$ of patients. Raw data for all aforementioned quality outcome indicators is presented in Table 1.

\section{Discussion}

While improvements in consenting practice were seen and maintained, it is notable that targeted interventions like stickers for risk documentation failed to produce significant change, with other measures such as copies being offered to patients slipping back. However, this analysis was limited by issues relating to the deployment of such pre-printed proformas and stickers. As mentioned previously, these interventions can be prone to attrition over time, and it was reported that supplies of the stickers were exhausted quickly with replacements reported as difficult to locate by junior colleagues, limiting their usage. Records audited later in cycle 2 showed a relative paucity of the stickers applied, although training on their content had also been delivered at time of the intervention, so the audit cycle was continued.

Further, as this project only focussed on patients with capacity, areas of poor practice may persist for those who do not. Given the demographic of patients admitted with hip fracture, many patients have comorbidities that can impair their mental capacity, either of irreversible aetiology such as dementia, or temporary such as delirium. Documentation of this process is longer and more time consuming, with the documentation used locally being several pages (29). Given that a formal assessment of capacity needs to be documented as well as any discussions (including procedure-specific risks) with family members or concerned parties, this is likely prone to the same pressures and issues causing poor practice outlined previously. Future work within the department will seek to review this documentation as well as considering specific interventions of benefit here.

Nevertheless, our experience from this improvement project finds that teaching sessions focussed on consenting and complications provided the greatest benefit, as well as developing newly rotated doctors understanding of the speciality. This would correspond as well with the recent recognition of the need for a personalised approach for consenting (as per Montgomery vs NHS Lanarkshire) and seemed to also generate cultural change in the department, with the usage of abbreviations continuing to decline without further input. From the literature, it has been demonstrated that pre-printed stickers and proformas can generate benefit and in our study, it would seem that some previous benefit was sustained with their 
introduction. However, it is notable that copies of forms being offered to patients declined significantly after the initial teaching session, which was not prompted for in the new hip fracture proforma. This may perhaps serve to highlight that such approaches, especially in the context of a busy admissions environment, can develop a degree of automaticity with other elements of the clerking and documentation process being neglected.

While further prompts within proformas may help to improve this, ultimately it is impossible to checklist the whole process and so these cannot serve to replace high quality training. Instead it would suggest that the role of proformas be limited and only be rolled out alongside detailed training on the condition they are covering. Without this, although consent forms may show thorough documentation of risks, the discussion between doctor and patient may be limited to merely reading off a list, rather than a detailed explanation. This is likely to be of increasing importance to consider as many trusts move to computerized admission records and digital consenting.

\section{Conclusions}

Errors or omission of important elements in documentation of patient consent can delay operations, expose hospital trusts to medico-legal risk and ultimately may represent a failure to fully respect patient autonomy. Our project sought to evaluate our current consenting practice for hip fractures and sought to identified recognized deficiencies in clarity of documentation, documentation of procedure appropriate risks and ensuring that patients were offered a copy of their consent form, trialling various methods to achieve this. While teaching sessions for the department brought about large improvements in our key process measures, the use of pre-printed stickers failed to significantly enhance this, and sharp deterioration was seen in areas that focus was taken away from. As digital consent and the use of preprepared documentation is set to expand, it is important to recognize the value of detailed training on consenting and procedure risks, so that not only is documentation of a high quality but also the patients actual understanding stemming from their conversation with the consenting doctor. This would therefore seem to require an ongoing personalized approach to consenting, which also reflects current legal thought in this area. Pre-printed checklists and proformas can be valuable tools to assist busy doctors, but their usage should be limited to adjuncts only and not be allowed to replace a high-quality discussion between physician and patient.

\section{Abbreviations}

$\mathrm{NHS}$

National Health Service

\section{Declarations}

\section{Ethics Approval and Consent to Participate}


As this involved a departmental audit of records following an already established intervention, formal ethical approval was not sought as per departmental protocol (not applicable).

\section{Consent for Publication}

Not Applicable

\section{Availability of Data and Material}

All data is stored securely within National Health Service information technology infrastructure. Please contact the corresponding author for access to data.

\section{Funding}

The authors have no external funding to report for this paper.

\section{Competing Interests}

None declared.

\section{Authors Contributions}

KS reviewed data gathered throughout the process, cleaned and analysed the data, re-drafted the submitted manuscript. He is guarantor. AA initiated the collaborative project and gathered the initial data. MB provided guidance and assisted in finalising the draft for submission. GNG provided project oversight.

\section{Acknowledgements}

All authors are grateful for the input and guidance provided by all members of the Trauma and Orthopaedics department in the Royal Gwent Hospital

\section{References}


1. NHFD 2018 annual report: Royal College of Physicians; 2018 [Available from: https://www.nhfd.co.uk/20/hipfractureR.nsf/docs/2018Report.

2. Moja L, Piatti A, Pecoraro V, Ricci C, Virgili G, Salanti G, et al. Timing matters in hip fracture surgery: patients operated within 48 hours have better outcomes. A meta-analysis and meta-regression of over 190,000 patients. PLoS One. 2012;7(10):e46175.

3. GMC. Consent: patients and doctors making decisions together 2008 [Available from: https://www.gmc-uk.org/ethical-guidance/ethical-guidance-for-doctors/consent.

4. Chong W, Fong ACY, Yeo YL, Ng APH, Neo EML, Sam VLE, et al. Reducing the number of invalid surgical consents in the day surgery unit. BMJ open quality. 2018;7(1):e000167.

5. Kentley J, Fox A, Taylor S, Hassan Y, Filipek A. The use of a pro forma to improve quality in clerking vascular surgery patients. BMJ Qual Improv Rep. 2016;5(1).

6. Beresford-Cleary NJ, Halliday J, Breusch SJ, Biant LC. Consent process for elective total hip and knee arthroplasty. Journal of orthopaedic surgery (Hong Kong). 2011;19(3):274-8.

7. Bajada S, Dwamena S, Abdul Z, Williams R, Ennis O. Improving consent form documentation and introduction of procedure-specific labels in a district general hospital. BMJ Qual Improv Rep. 2017;6(1).

8. Ahmad R, Shobaki S, Etezadi V, Raju S, Case R. Adequacy of consent in patients with distal radius fractures. International orthopaedics. 2009;33(5):1311-3.

9. Rahman L, Clamp J, Hutchinson J. Is consent for hip fracture surgery for older people adequate? The case for pre-printed consent forms. Journal of medical ethics. 2011;37(3):187-9.

10. Osborn GD, Pike H, Smith M, Winter R, Vaughan-Williams E. Quality of clinical case note entries: how good are we at achieving set standards? Annals of the Royal College of Surgeons of England. 2005;87(6):458-60.

11. DH. Good Practice in Consent Implementation Guide: Consent to Examination or Treatment: Department of Health; [Available from: http://www.dh.gov.uk/en/Publicationsandstatistics/.

12. Good Surgical Practice Domain 3: Communication, partnership and teamwork: Royal College of Surgeons of England; 2014 [Available from: https://www.rcseng.ac.uk/standards-andresearch/gsp/domain-3/.

13. Satyanarayana Rao KH. Informed consent: an ethical obligation or legal compulsion? Journal of cutaneous and aesthetic surgery. 2008;1(1):33-5.

14. Atrey A, Leslie I, Carvell J, Gupte C, Shepperd JA, Powell J, et al. Standardised consent forms on the website of the British Orthopaedic Association. The Journal of bone and joint surgery British volume. 2008;90(4):422-3.

15. Anderson $\mathrm{OA}$, Wearne IM. Informed consent for elective surgery-what is best practice? Journal of the Royal Society of Medicine. 2007;100(2):97-100.

16. Posner KL, Severson J, Domino KB. The role of informed consent in patient complaints: Reducing hidden health system costs and improving patient engagement through shared decision making. 
Journal of healthcare risk management : the journal of the American Society for Healthcare Risk Management. 2015;35(2):38-45.

17. Ho MY, Anderson AR, Nijjar A, Thomas C, Goenka A, Hossain J, et al. Use of the CRABEL Score for improving surgical case-note quality. Annals of the Royal College of Surgeons of England. 2005;87(6):454-7.

18. Agha R, Edison E, Fowler A. Improving the preoperative care of patients with femoral neck fractures through the development and implementation of a checklist. BMJ quality improvement reports. 2014;3(1):u202922.w1358.

19. Pucher PH, Aggarwal R, Qurashi M, Singh P, Darzi A. Randomized clinical trial of the impact of surgical ward-care checklists on postoperative care in a simulated environment. The British journal of surgery. 2014;101(13):1666-73.

20. Aveling EL, McCulloch P, Dixon-Woods M. A qualitative study comparing experiences of the surgical safety checklist in hospitals in high-income and low-income countries. BMJ Open. 2013;3(8):e003039.

21. Haynes AB, Weiser TG, Berry WR, Lipsitz SR, Breizat AH, Dellinger EP, et al. A surgical safety checklist to reduce morbidity and mortality in a global population. The New England journal of medicine. 2009;360(5):491-9.

22. Ng J, Abdelhadi A, Waterland P, Swallow J, Nicol D, Pandey S, et al. Do ward round stickers improve surgical ward round? A quality improvement project in a high-volume general surgery department. BMJ open quality. 2018;7(3):e000341.

23. Hale G, McNab D. Developing a ward round checklist to improve patient safety. BMJ Qual Improv Rep. 2015;4(1).

24. Barritt AW, Clark L, Teoh V, Cohen AM, Gibb PA. Assessing the adequacy of procedure-specific consent forms in orthopaedic surgery against current methods of operative consent. Annals of the Royal College of Surgeons of England. 2010;92(3):246-9; quiz 2p following 9.

25. St John ER, Scott AJ, Irvine TE, Pakzad F, Leff DR, Layer GT. Completion of hand-written surgical consent forms is frequently suboptimal and could be improved by using electronically generated, procedure-specific forms. The surgeon : journal of the Royal Colleges of Surgeons of Edinburgh and Ireland. 2017;15(4):190-5.

26. Hassen YAM, Johnston MJ, Singh P, Pucher PH, Darzi A. Key Components of the Safe Surgical Ward: International Delphi Consensus Study to Identify Factors for Quality Assessment and Service Improvement. Annals of surgery. 2018.

27. Williams DT, Hoare D, Shingler G, Fairweather C, Whitaker C. Data recording aids in acute admissions. International journal of health care quality assurance. 2013;26(1):6-13.

28. Chan SW, Tulloch E, Cooper ES, Smith A, Wojcik W, Norman JE. Montgomery and informed consent: where are we now? Bmj. 2017;357:j2224.

29. Patient Consent: Welsh Assembly Government; 2015 [Available from: http://www.wales.nhs.uk/sites3/page.cfm?orgid=465\&pid=11930. 


\section{Table}

Due to technical limitations, table 1 is only available as a download in the supplemental files section.

\section{Figures}

\section{CONSENT FOR FIXATION OF HIP FRACTURE:}

Infection, blood clots, pain, bleeding, wound problems, damage to blood vessels and nerves, leg length discrepancy, dislocation, impaired mobility, problems with bone healing, metal ware failure, further surgery, anaesthetic risk, death, risk of catheter or blood transfusion

\section{Figure 1}

Consent Sticker Scan

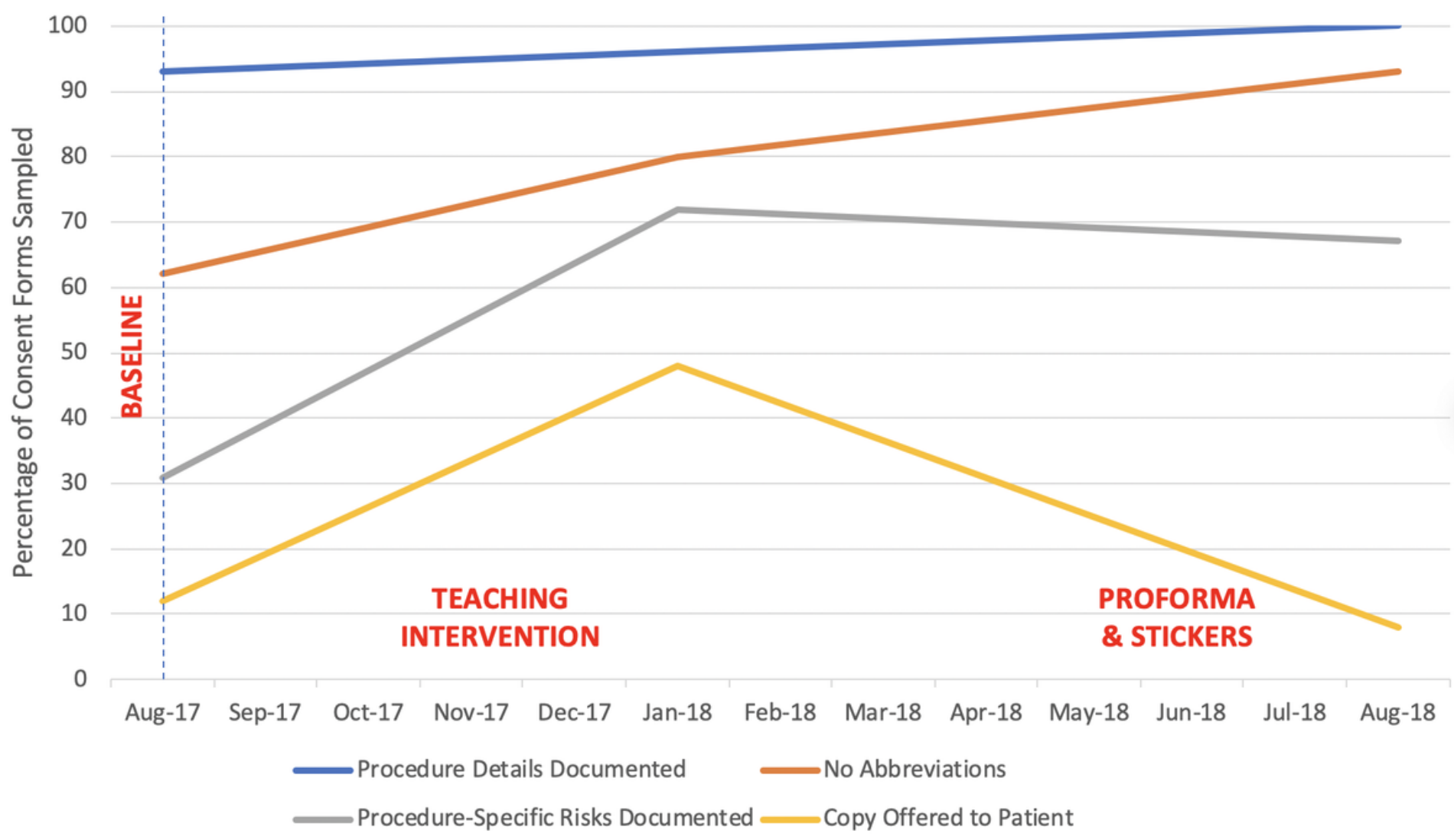

Figure 2

Runchart of Improvement Cycles

\section{Supplementary Files}

This is a list of supplementary files associated with this preprint. Click to download. 
- Table1QualityOutcomelndicators.xIsx 